\title{
ARTÍCULOS
}

Sometido 25.09.2018. Aprobado 04.06.2019

Evaluado por el sistema double blind review. Editor Científico: Diogo Henrique Helal

Versión original

DOI: http://dx.doi.org/10.1590/So034-759020190503

\section{LA FELICIDAD EN EL TRABAJO: VALIDACIÓN DE UNA ESCALA DE MEDIDA}

\author{
Happiness at work: Measurement scale validation \\ Felicidade no trabalho: Validação de uma escala de medição
}

JEL: C3; I31; M54

CAROLINA RAMIREZ-GARCIA ${ }^{1}$

cramgar@upo.es

ORCID: 0000-0002-7831-5139

\section{JUAN GARCÍA-ÁLVAREZ DE} PEREA ${ }^{1}$

jgaralv@upo.es

ORCID: 0000-0001-6432-0204

\section{JULIO GARCIA-DEL JUNCO²}

deljunco@us.es

ORCID: 0000-0003-0338-150X

1Universidad Pablo de Olavide, Departamento de Economía

Financiera y Contabilidad, Sevilla, España

2Universidad de Sevilla, Departamento de

Administración de Empresas y Marketing, Sevilla, España

\section{RESUMEN}

La felicidad del trabajador es un factor determinante de su eficiencia a corto y a largo plazo. En la literatura encontramos intentos de medir dicha felicidad directamente, y otros centrados en conocer cuáles son los elementos que generan esta felicidad. El presente trabajo realiza el análisis de validez de una escala propuesta por Del Junco, Espasandín, Dutschke y Palacios (2013) en la que se exponen factores determinantes de la felicidad del trabajador. El trabajo aporta una guía para la validación de escalas con un enfoque estructural y confirmatorio, basada en los datos de 262 empresas de la provincia de Sevilla (España). Asimismo, la escala muestra dos dimensiones (factores relacionados con el puesto de trabajo y factores relacionados con el trabajador) que confirman la necesidad de combinar la perspectiva del individuo y del entorno a la hora de analizar la felicidad laboral.

PALABRAS CLAVE | Felicidad en el trabajo, validación de escalas, análisis factorial exploratorio, análisis factorial confirmatorio, ecuaciones estructurales.

\section{ABSTRACT}

Workers' happiness is a determining factor of their short- and long-term efficiency. Several scholars have attempted to develop happiness measurement frameworks. The present work analyzes the validity of a scale proposed by Del Junco, Espasandin, Dutschke, and Palacios (2013) in which factors determining worker happiness are elucidated and examined. The work provides a guide for validating scales using a structural and confirmatory approach on the basis of data derived from 262 companies in the province of Seville (Spain). The scale examines two dimensions-factors related to the job and factors related to the worker-that confirm the need to combine both individual and environmental perspectives when analyzing worker happiness.

KEYWORDS I Happiness at work, scale validation, exploratory factor analysis, confirmatory factor analysis, structural equations.

\section{RESUMO}

A felicidade do trabalhador é um fator determinante de sua eficiência em curto e longo prazos. Na literatura, encontramos tentativas de medir a felicidade. O presente trabalho faz a análise da validade de uma escala proposta por Del Junco, Espasandín, Dutschke e Palacios (2013) na qual fatores que determinam a felicidade do trabalhador estão expostos. O trabalho fornece um guia para a validação de escalas com uma abordagem estrutural e confirmatória, com base em dados de 262 empresas na província de Sevilha (Espanha). Da mesma forma, a escala mostra duas dimensões (fatores relacionados ao trabalho e fatores relacionados ao trabalhador) que confirmam a necessidade de combinar a perspectiva do indivíduo e do ambiente ao analisar a felicidade no trabalho.

PALAVRAS-CHAVE / Felicidade no trabalho, validação de escalas, análise fatorial exploratória, análise fatorial confirmatória, equações estruturais. 


\section{INTRODUCCIÓN}

¿Qué hace a un trabajador feliz? La felicidad es una cuestión que ha sido abordada por áreas tan diversas como la Filosofía, la Literatura, la Psicología, la Sociología o la Antropología ente otras, pero es recientemente cuando surge con fuerza en el área de la Dirección de Empresas. Estamos ante un concepto del que es imposible ofrecer una definición única: no tiene una causa definida, sino que surge por la combinación de múltiples factores como pueden ser la genética, las características personales, el sexo, el nivel de estudios, las actividades realizadas, el entorno y un largo etcétera (Heller, Judge, \& Watson, 2002; Peterson, Park, Hall, \& Seligman, 2009).

En la literatura, la naturaleza multidimensional de la felicidad es clara, siendo puesta de manifiesto en los trabajos de Fisher (2014) y Rothmann (2013) y entre otros. Si bien las visiones hedónicas y eudaimónicas han centrado los orígenes de los trabajos de felicidad en distintos ámbitos, actualmente surge el concepto de flourishment (Diener et al. 2010) con el que se busca el origen del bienestar interior, de la salud mental, como base de la felicidad. Al ser complejo definir e influir sobre la percepción de felicidad, junto a los trabajos dedicados a medir la felicidad encontramos estudios centrados en analizar los antecedentes de la felicidad, factores que llevan al trabajador a una situación de bienestar.

Dado el elevado tiempo que se dedica al trabajo, la felicidad laboral supone un componente fundamental para desarrollar el bienestar personal y la felicidad (Fisher, 2014; Paschoal \& Tamayo, 2008). Si bien se ha demostrado que el trabajo aporta menos a la felicidad total de las personas comparativamente que la pareja, la familia, el ocio o los amigos, por el contrario, tiene un potencial de infelicidad altísimo y demostrado (Argyle, 1992). Así, la psicología positiva, centrada en el estudio del bienestar y la felicidad personal como emociones positivas, ha experimentado un gran auge dentro del campo de la felicidad en el trabajo (Fisher, 2010; Luthans, 2002; Seligman \& Csikszentmihalyi, 2000).

Del Junco, Espasandín, Dutschke y Palacios (2013), tras realizar una serie de entrevistas a trabajadores españoles y portugueses, desarrollan una escala de medida en el campo de la felicidad en el trabajo. Los autores, haciéndose eco de la falta de estudios sobre felicidad laboral en el área de Organización de Empresas, buscan proponer un instrumento accesible y útil para un directivo en su gestión cotidiana. El presente trabajo suministra dicho cuestionario a 262 empresas, describiendo el proceso de validación, depuración de datos, así como el análisis factorial para determinar las dimensiones subyacentes a la escala y la bondad de ajuste de la medida respecto a los datos reales del constructo a analizar.
A continuación, se expone cómo se ha introducido el término de felicidad en el trabajo revisando modelos propuestos previamente en la literatura, así como los antecedentes de la felicidad laboral. Seguidamente, se analizan las distintas evidencias de validez del cuestionario propuesto por Del Junco et al. (2013). Tras describir la metodología, se exponen y discuten los resultados principales del análisis. El artículo concluye con la exposición de las principales conclusiones, así como sus limitaciones y las futuras líneas de investigación derivadas de estas limitaciones.

\section{REVISIÓN DE LA LITERATURA}

El concepto felicidad depende del enfoque que adopta cada autor en su trabajo (Kiesebir \& Diener, 2008; Veenhoven, 1991), por lo que numerosos investigadores se refieren a él como "bienestar subjetivo" (Zelenski, Murphy, \& Jenkins, 2008). No obstante, este bienestar se ha reconocido como un componente de la felicidad, más rica, amplia y compleja que el bienestar (Diener, 2000; Diener, Suh, Lucas, \& Smith, 1999; Onusic, 2013). Numerosos trabajos concluyen que la felicidad surge de una medida subjetiva realizada por cada individuo acerca de los logros conseguidos en su propia vida (Diener, Sandvik, \& Pavot, 1991).

La felicidad es concebida desde una doble vertiente: por un lado (a) la visión hedónica, según la cual la felicidad vendrá determinada por el placer, la experiencia acumulada de afectos obtenidos, centrada principalmente en lo que la persona está sintiendo; y por otro lado (b) la perspectiva "eudaimónica" según la cual la felicidad es el grado de coherencia interna y de realización personal alcanzados, expresión de las capacidades potenciales individuales (Daniels, 2000; Ferreira, Silva, Fernandes, \& Almeida, 2008; Fisher, 2014; Van Horn, Taris, Schaufeli, \& Scheurs, 2004, Ryan \& Deci, 2001). Mientras que desde un punto de vista hedonista la felicidad es compatible con vivir una vida de valores superficiales, avaricia y explotación de otros, la perspectiva eudaimónica se centra en el contenido de la propia vida y los procesos implicados en "vivir bien" en lugar de "sentirse bien" (Rothmann, 2013). Tanto es así que se ha llegado a afirmar que la felicidad hedonista es insostenible a largo plazo en ausencia de un bienestar eudaimónico (Fisher, 2010).

La dualidad hedonista y eudaimónica se completa con el bienestar social (Fisher, 2014) así como con el concepto de flourishment, entendido como la experiencia de una vida que "va bien", una combinación de sentirse bien y funcionar efectivamente (Huppert \& So, 2013). Según Diener, el enfoque de flourishing incluye un propósito en la vida, la presencia de relaciones positivas, compromiso, competencia, autorrealización, 
optimismo y contribución al bienestar de otros (Diener et al. 2010). Se podría decir que si se estableciera un continuo en la salud mental, desde salud completa (flourishing) hasta falta de salud mental (languishing), la posición de las personas en ese continuo se determina por su puntuación en tres tipos de bienestar (emocional, psicológico y social) (Keyes, 2000). En aras de lograr este flourishing, Huppert y So (2013) diferencian diez características de la persona (competencia, estabilidad emocional, compromiso, sentido, optimismo, emociones positivas, relaciones positivas, resiliencia, autorrealización y vitalidad) recogiendo tanto aspectos hedónicos como eudamónicos. Por su parte, Keyes (2002) concreta esta salud mental en un conjunto de síntomas de sentimientos positivos y funcionamientos positivos en la vida.

La felicidad en el trabajo representa una cuestión de gran importancia, ya que la mayor parte de los seres humanos trabajan tanto por necesidad como por deseo: es una fuente no solo de ingresos, sino también de puesta en práctica de capacidades y habilidades personales, de enfrentar desafíos y de la propia realización personal (Moyano Díaz, Castillo Guevara, \& Lizana Lizana, 2008). Tanto es así que a quienes les gusta su trabajo no lo dejarían aun cuando no necesitaran el dinero (Argyle, 1992). Según Suh y Koo (2008) la felicidad laboral puede analizarse en el ámbito global del trabajador, en el organizativo o empresarial, o en el trabajo, centrándose la mayoría en la persona del trabajador (Groot \& Maassen van den Brink, 1999) o bien en el grupo o entorno en el que opera (Baker, Greenberg, \& Heemingway, 2006). Igualmente, este análisis se puede realizar considerando al trabajador como individuo soporte de procesos mentales (Judge Heller, \& Mount, 2002), o centrándose en el peso del “entorno" en la felicidad experimentada por el trabajador (Warr, 2013). Por último, una tercera diferenciación de enfoque es distinguir trabajos que miden la felicidad/bienestar del trabajador en sí, de aquellos que analizan los factores que llevan a esa percepción de felicidad.

Entre las medidas de felicidad en el trabajo existen diversidad de constructos dentro de los cuales el más utilizado es satisfacción laboral, encontrando otros como compromiso individual, compromiso organizativo, implicación laboral, motivación intrínseca, empuje y valor, afecto en el trabajo o resiliencia (Fisher, 2010). Ninguno tiene la capacidad para medir por sí solo la felicidad en el trabajo, por lo que lo más apropiado es tener en cuenta el conjunto de todos ellos para aproximarse al concepto final de felicidad en el trabajo (Fisher, 2010).

Esta naturaleza multidimensional de la felicidad es clara, y se pone de manifiesto explícitamente en los trabajos de Fisher (2014), Rothmann (2013) y Van Horn, et al. (2004) entre otros. Warr (1994) distingue cuatro dimensiones primarias (bienestar afectivo, aspiración, autonomía y competencia) y una quinta dimensión (funcionamiento integrado) que comprende las cuatro primarias y refleja a la persona como un todo. Por su parte, Van Horn et al. (2004) proponen cinco dimensiones, tres en línea con el trabajo de Warr (bienestar afectivo, social y profesional) e incluyen dos más (bienestar cognitivo y psicosomático). Paschoal y Tamayo (2008) proponen una escala de bienestar en el trabajo con una dimensión afectiva (emociones y humores en el trabajo) y una dimensión cognitiva (percepción de expresividad y realización personal en el trabajo). Según estos autores se diferencia entre bienestar subjetivo y bienestar psicológico, donde el primero adopta una visión hedónica, basada en la experiencia de placer frente a sufrimiento y el segundo un enfoque eudaimónico de la felicidad. Singh y Aggarwal (2018), por su parte, proponen cuatro dimensiones, dos organizativas: experiencias organizativas de apoyo, experiencias organizativas de no apoyo, y dos dimensiones del trabajador: flujo y motivación intrínseca y sentimientos repulsivos.

Fisher (2014), tratando de unificar las distintas perspectivas de felicidad, propone un modelo que tiene como núcleo el bienestar laboral, la experiencia de estados de ánimo y emociones placenteras mientras se trabaja. Posteriormente, incluye los estados de ánimo y las emociones negativas en el trabajo, los juicios de satisfacción en el trabajo y actitudes similares. Por último, su modelo se completa con las concepciones de bienestar general en el trabajo, los componentes eudaimónicos y bienestar social.

En cuanto a trabajos que estudian factores que originan la felicidad laboral, puede decirse que esta viene explicada por una suma de características del trabajo, como el salario, la posibilidad de promoción, el horario, el nivel de peligrosidad del puesto o su monotonía, etc.; del entorno del trabajo, como el ambiente de la empresa, el salario medio en comparación con el propio, la dimensión de la empresa y su potencial, etc.; así como del trabajador, su edad, sexo, nivel de estudios, situación sentimental, etc. (Linz \& Semykina, 2012). Igualmente, Parker y Hyett (2011) reconocen el respeto organizativo por el empleado, el cuidado del empleado y la intrusión del trabajo en la vida privada como antecedentes positivos y negativos del bienestar (Fisher, 2014).

Una variable frecuentemente analizada como fuente de felicidad laboral ha sido el salario. Algunos autores defienden que elevados ingresos contribuyen a un aumento de la felicidad personal (Clark, Kristensen, \& Westergård-Nielsen, 2009), mientras que otros muestran que esta relación dependerá de la percepción de los trabajadores acerca de lo equitativa que consideran esa contraprestación a su trabajo (Sloane \& Williams, 2000). Judge, Piccolo, Podsakoff, Shaw y Rich (2010) de forma aislada no encontraron efectos del salario sobre la satisfacción laboral, pero observaron que los empleados con mayores salarios estaban ligeramente más satisfechos que los que ganan menos. 
Para Warr (2013) los salarios tienen una influencia positiva en trabajadores con bajos ingresos, comportándose de forma neutra a partir de cierto nivel de ingresos. En esta línea encontramos otra corriente de estudios que consideran las ganancias intrínsecas, como la capacidad de adquirir nuevas habilidades y su efecto positivo en el nivel de felicidad alcanzado, como elemento generador de felicidad (Origo \& Pagani, 2009).

La estabilidad profesional, reflejada en la calidad del contrato laboral, en su carácter definido o indefinido, también ha sido analizada frecuentemente como antecedente de la felicidad. Sanín, López y Gómez (2015) y Useche y Parra (2002) encuentran una notable influencia de esta estabilidad laboral en la felicidad del trabajador. Por su parte, Hosie y Sevastos (2009) y Sanín y Restrepo (2009) observan que la falta de garantía en la permanencia del puesto de trabajo provoca una sensación negativa que limita la felicidad del trabajador. Así, la persona en la organización manifestará una mayor satisfacción en la medida que se sienta seguro profesionalmente (Wright, Larwood, \& Denney, 2002), existiendo una relación negativa entre los contratos más precarios con el nivel de felicidad del trabajador (Gamero, 2007). Pero no solo es la estabilidad profesional la que influye en el nivel de felicidad del trabajador, ya que la “estabilidad interior" también afecta a dicha felicidad tanto en el ámbito laboral como en otras áreas de la vida personal (Judge et al., 2002).

Encontramos finalmente otros estudios que parecen poner el énfasis en el grado de independencia y libertad en el trabajo, más allá de las ganancias o la forma contractual. Así, Benz y Frei (2004) observan un mayor grado de felicidad en aquellos trabajadores que optan por el autoempleo, debido a que la sensación de libertad que experimentan -no que necesariamente tengan- es mayor. Como sucedía con el efecto del nivel salarial, Warr (2013) vuelve a encontrar que esta autonomía afecta positivamente a la felicidad en niveles de autonomía menores y de forma neutral a partir de cierto valor. Ahora bien, si la autonomía crece excesivamente los valores de felicidad laboral alcanzados pueden verse reducidos.

Respecto a antecedentes del flourishment, el modelo Warr (1987) reconoce nueve condiciones ambientales (oportunidad de control, uso de habilidades, contacto interpersonal, exigencias de objetivos y tareas, variedad, claridad ambiental, disponibilidad de dinero, seguridad física y posición social) responsables del bienestar psicológico. Igualmente, el modelo de las características del trabajo (Hackman \& Oldham, 1980) especifica cinco dimensiones de una tarea (variedad de habilidades, identidad de la tarea, significado de la tarea, responsabilidad-autonomía y comentarios) que pueden afectar la satisfacción laboral, el compromiso laboral y a otros aspectos del flourishment. Smith, Kendall y Hulin (1969) en esta línea postularon cinco facetas de la satisfacción laboral: trabajo, supervisión, compañeros de trabajo, remuneración y ascensos

\section{DESCRIPCIÓN DE LA ESCALA Y RECOPILACIÓN DE DATOS}

En el trabajo de Del Junco et al. (2013), los autores se proponen extraer una escala de medida de la felicidad ante las respuestas a tres interrogantes:

- ¿ ¿Cuál es su definición de felicidad?

- ¿Qué entiende por felicidad en la organización? y

- ¿Qué es felicidad en el trabajo? (Del Junco et al., 2013, p. 10).

Así, mediante entrevistas abiertas a trabajadores portugueses y españoles, se realizaron estas preguntas, cuya transcripción se analizó con la herramienta ATLAS / TI V6.0 llevándose a cabo un análisis de contenido que permitió extraer los factores más relevantes. Estos factores dieron, así, lugar a la propuesta de una serie de ítems para medir felicidad organizacional (Del Junco et al., 2013, p. 15) estructurada en 15 ítems (ver Cuadro 1). Previamente a estas cuestiones, se realizan dos preguntas de control sobre edad y sexo que corresponden a variables individuales que la literatura considera que pueden afectar al nivel de felicidad.

Cuadro 1. Cuestionario propuesto por Del Junco et al. (2013)

\begin{tabular}{c|l}
\hline \multicolumn{2}{l}{ Evalúe del 1 al 7 las siguientes afirmaciones } \\
\hline 1 & Disfruto con mi trabajo \\
\hline 2 & La familia me aporta felicidad \\
\hline 3 & Tengo buena salud \\
\hline 4 & En mi vida el amor ocupa un lugar importante \\
\hline 5 & Tengo estabilidad interior \\
\hline 6 & Tengo bienestar objetivo \\
\hline 7 & Tengo estabilidad profesional \\
\hline 8 & En el trabajo obtengo las justas recompensas \\
\hline 9 & El clima organizacional de la empresa es bue \\
\hline 10 & Los jefes dirigen bien \\
\hline 11 & Disfruto haciendo bien mi trabajo \\
\hline 12 & El clima organizacional en mi unidad de trabajo es bueno \\
\hline 13 & La motivación interna de mi puesto es alta \\
\hline 14 & Mis tareas en la empresa están bien diseñadas \\
\hline 15 & Soy extrovertido/a \\
\hline
\end{tabular}


Con el objetivo de validar esta escala, para el presente trabajo se seleccionaron empresas representativas de distintos tamaños y sectores de actividad de la provincia de Sevilla recogidas en el censo suministrado por "einforma.com" (Ver Ficha Técnica en el Cuadro 2).

\section{Cuadro 2. Ficha técnica de la encuesta}

\begin{tabular}{l|l}
\hline $\begin{array}{l}\text { Método recolección } \\
\text { datos }\end{array}$ & $\begin{array}{l}\text { Directo, con presencia en las empresas } \\
\text { participantes. }\end{array}$ \\
\hline Ámbito geográfico & Provincia de Sevilla \\
\hline Universo & $\begin{array}{l}\text { Micro, pequeñas, medianas y grandes } \\
\text { empresas }\end{array}$ \\
\hline Número de encuestas & 262 \\
\hline
\end{tabular}

Los cuestionarios se realizaron visitando a las empresas para maximizar el número de respuestas. El primer contacto era con el responsable de la empresa, preferiblemente del ámbito de recursos humanos, mostrando nuestro deseo de que participase en nuestra investigación. Las características de los individuos encuestados son las recogidas en la Tabla 1:

Tabla 1. Composición de la muestra

\begin{tabular}{c|c}
\hline \multicolumn{2}{c}{ Sexo } \\
\hline Mujeres & $39,40 \%$ \\
\hline Hombres & $60,60 \%$ \\
\hline \multicolumn{2}{|c}{ Edad } \\
\hline $30-29$ & $12,40 \%$ \\
\hline $40-49$ & $35,30 \%$ \\
\hline $50-59$ & $24,70 \%$ \\
\hline $60-69$ & $22,90 \%$ \\
\hline mayores de 70 & $4,70 \%$ \\
\hline
\end{tabular}

\section{Análisis de las evidencias de validez de la escala}

La validez de un instrumento de medida para un uso concreto es definida como el grado en el que la evidencia y la teoría soportan la interpretación del instrumento de investigación para ese uso determinado (AERA, APA, NCME, 2014). La validación consistirá, así, en obtener evidencia empírica, examinar la literatura relevante y llevar a cabo análisis lógicos para evaluar cada proposición. A diferencia de la distinción tradicional de Validez de Contenido, de Constructo y de Criterio, en la actualización de los Estándares de la American Education Research Association (AERA), American Psychological Association (APA) y el National Council on Measurement in Education de 2014, se propone el concepto de validez como único y manifestado en evidencias de validez (AERA, APA, NCME, 2014, pp.14). Dichas evidencias de validez se pueden concretar en:

- $\quad$ Evidencias basadas en el test de contenido: Obtenidas del análisis de la relación entre el contenido del cuestionario y el constructo teórico que se trata de medir.

- Evidencias basadas en el proceso de respuesta: Análisis teóricos y empíricos del proceso de respuesta que pueden dar evidencia del ajuste del constructo y la respuesta dada al test.

- Evidencias basadas en la estructura interna: Indica el grado en el que las relaciones entre los ítems del test y los componentes del test conforman el constructo que se quiere interpretar.

- Evidencia basada en la relación con otras variables: La interpretación de un uso dado del test implica que el constructo debe estar relacionado con otras variables.

- $\quad$ Evidencias convergente y discriminante: En el primer caso hablamos de la relación entre las puntuaciones del test y otras medidas que tratan de evaluar el mismo constructo o similar, mientras que la relación entre puntuaciones del test y medidas con diferentes propósitos da la evidencia discriminante.

A continuación, analizamos dichas evidencias respecto a la escala propuesta por Del Junco et al. (2013).

\section{Evidencias basadas en el test de contenido}

Para obtener este tipo de evidencias es necesario concretar el constructo teórico que se trata de medir, entendiendo por constructo el concepto o las características que se quieren evaluar con el test diseñado (AERA, APA, NCME, 2014). La descripción detallada de este constructo proporciona un marco conceptual para el instrumento de medida. En el caso de la escala propuesta por Del Junco et al. (2013) en primer lugar se analiza en qué medida los ítems reflejan las vertientes teóricas del concepto 
felicidad descritas en el apartado teórico de este trabajo. Para ello, hemos clasificado los ítems del cuestionario como "Hed" en caso de que reflejen la vertiente hedónica de la felicidad y "Eud" en caso de reflejar su vertiente eudaimónica (Fisher, 2010). Igualmente, dado que los factores que influyen en la felicidad del trabajador son una conjunción de aspectos individuales y del entorno (Warr, 2013), la segunda columna del Cuadro 3 clasifica las afirmaciones en función de estos aspectos.

\section{Cuadro 3. Evidencia de validez de contenido}

\begin{tabular}{|c|c|c|c|}
\hline & $\begin{array}{l}\text { Evalúe del } 1 \text { al } 7 \text { las siguientes } \\
\text { afirmaciones }\end{array}$ & $\mathrm{Hed} /$ Eud $^{1}$ & Ind/Ent ${ }^{2}$ \\
\hline 1 & Disfruto con mi trabajo & Eud & Ind \\
\hline 2 & La familia me aporta felicidad & Hed & Ent \\
\hline 3 & Tengo buena salud & Hed & Ind \\
\hline 4 & $\begin{array}{l}\text { En mi vida el amor ocupa un lugar } \\
\text { importante }\end{array}$ & Hed & Ind \\
\hline 5 & Tengo estabilidad interior & Hed & Ind \\
\hline 6 & Tengo bienestar objetivo & Hed & Ind \\
\hline 7 & Tengo estabilidad profesional & Hed & Ent \\
\hline 8 & $\begin{array}{l}\text { En el trabajo obtengo las justas } \\
\text { recompensas }\end{array}$ & Eud & Ent \\
\hline 9 & $\begin{array}{l}\text { El clima organizacional de la } \\
\text { empresa es bueno }\end{array}$ & Eud & Ent \\
\hline 10 & Los jefes dirigen bien & Eud & Ent \\
\hline 11 & Disfruto haciendo bien mi trabajo & Eud & Ind \\
\hline 12 & $\begin{array}{l}\text { El clima organizacional en mi } \\
\text { unidad de trabajo es bueno }\end{array}$ & Eud & Ent \\
\hline 13 & $\begin{array}{l}\text { La motivación interna de mi puesto } \\
\text { es alta }\end{array}$ & Eud & Ind \\
\hline 14 & $\begin{array}{l}\text { Mis tareas en la empresa están } \\
\text { bien diseñadas }\end{array}$ & Eud & Ent \\
\hline 15 & Soy extrovertido/a & Hed & Ind \\
\hline
\end{tabular}

Notas: ${ }^{1}$ Cuestión que aborda el componente Hedónico o Eudaimónico.

${ }^{2}$ Cuestión que plantea un factor del Individuo o del Entorno.

Los ítems extraídos del análisis de contenido reflejan tanto factores relacionados con el trabajador (Groot \& Maassen van den Brink, 1999) como del grupo en el que desarrolla su tarea (Baker et al., 2006; Suh \& Koo, 2008). Al ser un cuestionario extraído de un estudio exploratorio, estos ítems no reflejan estrictamente variables delimitadas previamente por el investigador, sino que muestra las variables que los entrevistados perciben como relevantes ante las preguntas del campo de la felicidad laboral que realizaron los entrevistadores. Observando dichas variables podemos afirmar que el contenido de los ítems se encuadra con la vertiente de trabajos que tratan antecedentes de felicidad, no con medidas directas de la felicidad.

\section{Evidencias de validez en el proceso de respuesta}

Los cuestionarios presentan ciertas limitaciones que el investigador puede controlar si sigue los principios fundamentales del diseño y la administración de cuestionarios (Dillman, 2000). En esta categoría se encuentran los procedimientos de pretest, de seguimiento y el análisis del sesgo de no respuesta. En el caso de la escala propuesta por Del Junco et al. (2013) se analizó el sesgo de no respuesta tanto para el sector como para el tamaño de la empresa, para comprobar si la muestra podía generar errores a la hora de validar la escala. La prueba no paramétrica de Chi cuadrado mostró que la composición de la muestra reflejaba la composición de la población.

\section{Evidencias basadas en la estructura interna}

Para analizar la consistencia de los ítems de la escala en uno o varios factores, se contrasta en primer lugar la normalidad de los datos y a continuación se realiza un Análisis Factorial Exploratorio (AFE) usando el programa estadístico SPSS 22 (George \& Mallery, 2003) para contrastar su multidimensionalidad y el nivel de fiabilidad (Hair, Anderson, Tatham, \& Black, 1999). Para depurar los casos atípicos o outliers que pueden afectar a las conclusiones de validez, se usó el intervalo intercuartílico sustituyendo los datos que salen de ese intervalo por la media de los valores de la variable una vez eliminado el outlier. Un ejemplo para una de las variables del estudio sería el que se recoge en la Tabla 2.

Tabla 2. Cálculo del intervalo detección valores atípicos

\begin{tabular}{c|c}
\hline Variable 1 & Disfruto de mi trabajo \\
\hline Mediana (Q2) & 0,365 \\
\hline Rango IQ & 1,349 \\
\hline Q1 & $-0,309$ \\
\hline Q3 & 1,04 \\
\hline Lim Inf ${ }^{1}$ & $-4,358$ \\
\hline $\operatorname{Lim~sup}^{1}$ & 5,089 \\
\hline
\end{tabular}

Nota: 'Límites del intervalo fuera del cual se consideran a los valores atípicos o outliers. 
La normalidad de los datos se analizó mediante la prueba de Kolmogorov-Smirnoff, que mostró que los datos no siguen una distribución normal, usándose estadísticos asintóticamente robustos, no afectados por la no normalidad de los datos. En el Análisis Factorial Exploratorio (AFE) se exige a las distintas dimensiones un Alfa de Cronbach que supere el o,7 como criterio de fiabilidad (Hair et al., 1999). Respecto a las medidas de Varianza Extraída (varianza que es capaz de explicar el factor o variable latente) y Fiabilidad Compuesta (consistencia interna de los indicadores), aunque tradicionalmente se han establecido como puntos de corte 0,5 y 0,7 respectivamente (Hair et al., 1999), Valentini y Damásio (2017) afirman que no se puede fijar un umbral estricto para estas medidas, ya que sufren alteraciones en función del número de ítems y de la homogeneidad de las cargas factoriales y podría limitar la interpretación de los resultados (Valentini \& Damásio, 2017). Por esto, dichos valores son analizados con cautela. El AFE fue realizado con el método de extracción de máxima verosimilitud, que ofrece resultados cercanos a los del Análisis Factorial Confirmatorio (AFC) y rotación varimax por la facilidad que ofrecen a la hora de la interpretación de las cargas factoriales (Hair et al., 1999). La significatividad del modelo se analiza con el coeficiente KMO (Kaiser-Meyer-Olkin), cuyos valores deben ser próximos a la unidad, así como con la prueba de esfericidad de Barlett, que debe ser significativa (Hair et al., 1999).

El primer resultado que obtenemos de este AFE es el recogido en la Tabla 3, donde el valor de la medida KMO de 0.876 indica una correlación alta de las variables, considerándose el test como notable (sería muy bueno en casos de $K M>0,9$ ) y una prueba de esfericidad de Barlett significativa, justificando así el análisis factorial. La prueba factorial muestra que en la escala se pueden distinguir dos factores que explican un total de $45,53 \%$ de la varianza, donde la matriz factorial carga de la siguiente forma:

\section{Tabla 3. Matriz factorial inicial de la escala}

\begin{tabular}{c|l|c|c}
\hline & Redacción Item & Factor $\mathbf{1}$ & Factor 2 \\
\hline 1 & Disfruto con mi trabajo & 0,616 & 0,288 \\
\hline 2 & La familia me aporta felicidad & 0,09 & 0,508 \\
\hline 3 & Tengo buena salud & 0,03 & 0,504 \\
\hline 5 & $\begin{array}{l}\text { En mi vida el amor ocupa un lugar } \\
\text { importante }\end{array}$ & 0,06 & 0,398 \\
\hline 6 & Tengo estabilidad interior & 0,19 & 0,756 \\
\hline 7 & Tengo estabilidad profesional & 0,459 & 0,322 \\
\hline 8 & $\begin{array}{l}\text { En el trabajo obtengo las justas } \\
\text { recompensas }\end{array}$ & 0,748 & 0,085 \\
\hline
\end{tabular}

Tab

\begin{tabular}{|c|c|c|c|}
\hline & Redacción Item & Factor 1 & Factor 2 \\
\hline 9 & $\begin{array}{l}\text { El clima organizacional de la } \\
\text { empresa es bueno }\end{array}$ & 0,834 & $-0,018$ \\
\hline 10 & Los jefes dirigen bien & 0,892 & 0,091 \\
\hline 11 & Disfruto haciendo bien mi trabajo & 0,292 & 0,58 \\
\hline 12 & $\begin{array}{l}\text { El clima organizacional en mi } \\
\text { unidad de trabajo es bueno }\end{array}$ & 0,668 & 0,229 \\
\hline 13 & $\begin{array}{l}\text { La motivación interna de mi } \\
\text { puesto es alta }\end{array}$ & 0,664 & 0,35 \\
\hline 14 & $\begin{array}{l}\text { Mis tareas en la empresa están } \\
\text { bien diseñadas }\end{array}$ & 0,708 & 0,214 \\
\hline 15 & Soy extrovertido/a & 0,327 & 0,257 \\
\hline
\end{tabular}

En la Tabla 3 podemos observar que la variable número "En mi vida el amor ocupa un lugar importante" tiene cargas factoriales sensiblemente inferiores a 0,5 , lo que supone indicios de problemas de fiabilidad. Asimismo, el Alpha de Cronbach de la escala, si se elimina esta variable, pasa de 0,884 a o,888, por lo que consideramos relevante eliminar la variable de la escala definitiva. Lo mismo sucede con las variables "Tengo buena salud", "La familia me aporta felicidad” y "Soy Extrovertido", que tras su eliminación el AF queda de la forma indicada en la Tabla 4:

\section{Tabla 4. Matriz factorial final de la escala}

\begin{tabular}{|c|c|c|}
\hline Redacción Item & Factor 1 & Factor 2 \\
\hline $\begin{array}{l}\text { En el trabajo obtengo las justas } \\
\text { recompensas }\end{array}$ & 0,699 & 0,262 \\
\hline $\begin{array}{l}\text { El clima organizacional de la empresa es } \\
\text { bueno }\end{array}$ & 0,856 & 0,085 \\
\hline Los jefes dirigen bien & 0,871 & 0,24 \\
\hline $\begin{array}{l}\text { El clima organizacional en mi unidad de } \\
\text { trabajo es bueno }\end{array}$ & 0,613 & 0,349 \\
\hline La motivación interna de mi puesto es alta & 0,555 & 0,539 \\
\hline $\begin{array}{l}\text { Mis tareas en la empresa están bien } \\
\text { diseñadas }\end{array}$ & 0,649 & 0,35 \\
\hline Disfruto con mi trabajo & 0,504 & 0,502 \\
\hline Tengo estabilidad interior & 0,106 & 0,627 \\
\hline Tengo bienestar objetivo & 0,308 & 0,574 \\
\hline Tengo estabilidad profesional & 0,353 & 0,476 \\
\hline Disfruto haciendo bien mi trabajo & 0,138 & 0,716 \\
\hline
\end{tabular}


La eliminación de estos ítems, desde una perspectiva de análisis del constructo, puede responder a que trata de factores teóricamente relacionados con la felicidad (amor, salud, familia, carácter extrovertido), pero sin una relación clara con la felicidad laboral que se estudia. A la vista de los resultados, y analizando el contenido de los ítems de cada dimensión, podemos distinguir una primera dimensión de factores relacionados con el puesto de trabajo (Alpha de Cronbach del 0,91) y otra dimensión que recoge factores del trabajador (Alpha de Cronbach del 0,72).

Estas escalas son útiles para la práctica profesional, donde la puntuación se usa para tener una orientación de la situación de felicidad laboral de los empleados. No obstante, con un enfoque más académico para utilizar la escala en modelos de estudio más complejos, se lleva a cabo el Análisis Factorial Confirmatorio (AFC) mediante modelos de Ecuaciones Estructurales (Bagozzi, Yi, \& Phillips, 1991), determinando así qué variables formarían la escala de medida definitiva.
El enfoque de ecuaciones estructurales introduce la estimación del error de medida de los factores y con ésta las medidas de bondad de ajuste que estudian la validez de las ecuaciones de regresión y la cercanía del modelo teórico con la realidad (Byrne, 2006). Estas medidas de bondad de ajuste se basan en la comparación de la matriz de covarianzas de la muestra con la de la población, mediante el estadístico Chi-Cuadrado y su $p$-valor asociado. Dado que este estadístico es sensible al tamaño muestral y está basado en la centralidad de su distribución, en las últimas décadas han surgido numerosos índices de ajuste alternativos con una visión más pragmática (Byrne, 2006). Para la no normalidad de los datos se utilizan indicadores asintóticamente robustos, entre los que destaca la Chi Cuadrado Satorra-Bentler (Satorra, 1993), estadístico más fiable para evaluar la estructura de medias y covarianzas bajo varias distribuciones y tamaños muestrales (Hu, Bentler, \& Kano, 1992; Curran, West, \& Finch, 1996). En la Tabla 5 se resumen los índices de ajuste más frecuentes a analizar junto a la Chi-Cuadrado ${ }_{S B}$ y su P valor,

\section{Tabla 5. Medidas de bondad de ajuste}

\begin{tabular}{|c|c|c|c|}
\hline \multicolumn{2}{|c|}{ Índice de ajuste } & \multirow{2}{*}{$\begin{array}{c}\text { Valor de } \\
\text { referencia } \\
\text { >0,95 }\end{array}$} & \multirow{2}{*}{$\begin{array}{l}\text { Comentario } \\
\text { lemas de muestras pequeñas y, aunque un valor del } \\
\text { o originalmente adecuado (Bentler, 1992), se ha } \\
\text { a o,95 (Hu \& Bentler, 1999). El CFI es considerado } \\
\text { (Byrne, 2006). }\end{array}$} \\
\hline 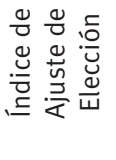 & $\begin{array}{l}\text { Comparative Fit } \\
\text { Index (CFI) }\end{array}$ & & \\
\hline \multirow{3}{*}{ 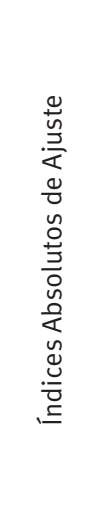 } & $\begin{array}{l}\text { Goodness of Fit } \\
\text { Index (GFI) }\end{array}$ & $>0.9$ & $\begin{array}{l}\text { Considerado análogo al valor del R cuadrado de los modelos de regresión múltiple (Tanaka, } \\
\text { 1993). }\end{array}$ \\
\hline & $\begin{array}{l}\text { Adjusted } \\
\text { Goodness of fit } \\
\text { Index (AGFI) }\end{array}$ & $>0,9$ & $\begin{array}{l}\text { Ajustado para los grados de libertad del modelo, penalizando la incorporación de } \\
\text { parámetros adicionales }\end{array}$ \\
\hline & $\begin{array}{l}\text { McDonald Fit } \\
\text { Index (MFI) }\end{array}$ & $>0,89$ & Hu y Bentler (1999) concretaron el valor de corte para MFI distinto al del GFI y AGFI \\
\hline 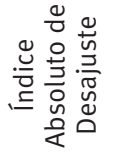 & $\begin{array}{l}\text { Root mean } \\
\text { square error of } \\
\text { approximation } \\
\quad \text { (RMSEA) }\end{array}$ & $<0,6$ & $\begin{array}{l}\text { Es uno de los criterios que más información da en el modelo de estructura de covarianzas } \\
\text { (Byrne, 2006). Sensible a los errores de especificación del modelo, por poder usarse como } \\
\text { guía interpretativa acerca de la calidad del modelo y por permitir construir intervalos de } \\
\text { confianza alrededor de los valores del RMSEA }\end{array}$ \\
\hline
\end{tabular}


En el caso de la dimensión referente a las características del puesto de trabajo, su primer análisis, como muestra la Tabla 6 , proporciona medidas de bondad de ajuste aceptables que en algunos indicadores no cumplen con los límites exigibles:

Tabla 6. Medidas de bondad de ajuste iniciales del factor 1

\begin{tabular}{l|c|c}
\hline & $\begin{array}{c}\text { Valores } \\
\text { de referencia }\end{array}$ & $\begin{array}{c}\text { Características del } \\
\text { puesto de trabajo }\end{array}$ \\
\hline $\mathrm{X}_{\text {SB }}$ (g.l.) & $>0,05$ & $39,67(9)$ \\
\hline P-Valor & $>0,9$ & 0 \\
\hline $\mathrm{GFI}$ & $>0,9$ & 0,932 \\
\hline $\mathrm{AGFI}$ & $<0,6$ & 0,841 \\
\hline RMSEA ${ }_{\text {robusto }}$ & $>0,95$ & 0,114 \\
\hline CFI ${ }_{\text {robusto }}$ & $>0,89$ & 0,941 \\
\hline MFI & $>0,7$ & 0,943 \\
\hline Alpha de Cronbach & $>0,7$ & 0,893 \\
\hline Fiabilidad Compuesta & $>0,5$ & 0,894 \\
\hline Varianza Extraída & & 0,588 \\
\hline
\end{tabular}

Partiendo de que la Chi-Cuadrado ${ }_{S B}$ y su P valor no son significativos, debemos buscar qué variable(s) motiva(n) esta falta de ajuste del modelo apoyándonos en el Test de Multiplicadores de Lagrange (LMTest) que indica qué relaciones se pueden incluir para mejorar el ajuste del modelo. Según este test, correlacionar la variable "Mis tareas en la empresa están bien diseñadas" con las variables "En el trabajo obtengo las justas recompensas" y "La motivación de mi puesto es alta”, se logra un mejor ajuste del modelo.

\section{Tabla 7. Medidas de bondad de ajuste del factor 1 con factores correlacionados}

\begin{tabular}{l|c|c}
\hline & $\begin{array}{c}\text { Valores } \\
\text { de referencia }\end{array}$ & $\begin{array}{c}\text { Características del } \\
\text { puesto de trabajo }\end{array}$ \\
\hline $\mathrm{X}_{\mathrm{SB}}{ }^{\text {(g.l.) }}$ & $>0,05$ & $6,95(6)$ \\
\hline P-Valor & $>0,9$ & 0,325 \\
\hline $\mathrm{GFI}$ & $>0,9$ & 0,987 \\
\hline $\mathrm{AGFI}$ & $<0,6$ & 0,956 \\
\hline RMSEA & $>0,95$ & 0,025 \\
\hline CFI & $>0,89$ & 0,998 \\
\hline robusto & $>0,7$ & 0,998 \\
\hline Alpha de Cronbach & $>0,7$ & 0,893 \\
\hline Fiabilidad Compuesta & $>0,5$ & 0,901 \\
\hline Varianza Extraída & & 0,606 \\
\hline
\end{tabular}
aconsejable eliminar el ítem que sea fuente de este problema (en este caso, la variable 14) logrando así un buen ajuste del modelo, como se muestra en la Tabla 8.

Tabla 8. Medidas de bondad de ajuste del factor 1 eliminando la variable 14

\begin{tabular}{l|c|c}
\hline & $\begin{array}{c}\text { Valores } \\
\text { de referencia }\end{array}$ & $\begin{array}{c}\text { Características del } \\
\text { puesto de trabajo }\end{array}$ \\
\hline $\mathrm{X}_{\text {SB }}^{2}$ (g.l.) & $>0,05$ & $6,84(5)$ \\
\hline P-Valor & $>0,9$ & 0,23 \\
\hline GFI & $>0,9$ & 0,986 \\
\hline AGFI & $\iota 0,6$ & 0,959 \\
\hline RMSEA robusto & $>0,95$ & 0,038 \\
\hline CFI robusto & $>0,89$ & 0,995 \\
\hline MFI & $>0,7$ & 0,996 \\
\hline Alpha de Cronbach & $>0,7$ & 0,876 \\
\hline Fiabilidad Compuesta & $>0,5$ & 0,879 \\
\hline Varianza Extraída & & 0,597 \\
\hline
\end{tabular}

Los datos de bondad de ajuste de partida para la escala referente a las características internas del trabajador son los recogidos en la Tabla 9:

Tabla 9. Medidas de bondad de ajuste iniciales del factor 2

\begin{tabular}{l|c|c}
\hline & $\begin{array}{c}\text { Valores } \\
\text { de referencia }\end{array}$ & $\begin{array}{c}\text { Características del } \\
\text { puesto de trabajo }\end{array}$ \\
\hline $\mathrm{X}_{\text {SB }}^{2}$ (g.l.) & $>0,05$ & $30,65(5)$ \\
\hline P-Valor & $>0,9$ & 0 \\
\hline GFI & $>0,9$ & 0,933 \\
\hline AGFI & $<0,6$ & 0,799 \\
\hline RMSEA ${ }_{\text {robusto }}$ & $>0,95$ & 0,14 \\
\hline CFI ${ }_{\text {robusto }}$ & $>0,89$ & 0,869 \\
\hline MFI & $>0,7$ & 0,952 \\
\hline Alpha de Cronbach & $>0,7$ & 0,761 \\
\hline Fiabilidad Compuesta & $>0,5$ & 0,749 \\
\hline Varianza Extraída & & 0,429 \\
\hline
\end{tabular}

Si bien las medidas de bondad de ajuste no se encuadran en los intervalos ideales, en este caso se opta por no seguir ajustando esta dimensión, ya que los cuatro ítems de partida con los que se cuentan hacen muy probable que tengamos problemas de especificación del modelo si se eliminan variables. 


\section{Evidencias convergente y discriminante}

En este paso se trata de confirmar que cada factor extraído del análisis factorial confirmatorio mide un solo constructo, y que dicho constructo es significativamente distinto del resto de los factores recogidos en el cuestionario. De esta forma se estudia si las escalas miden solo su constructo objetivo, o, por el contrario, se confunden con otros factores del estudio (Sánchez \& Sarabia, 1999). Para ofrecer evidencias de este tipo seguimos el procedimiento propuesto por Fornell y Larcket (1981): existe evidencia discriminante siempre y cuando la varianza extraída sea superior a las correlaciones entre dos constructos elevadas al cuadrado. Esta comprobación se suele concretar en una matriz en la que se compara la correlación que guardan los factores dos a dos con la varianza extraída de cada factor. En nuestro caso la matriz es sumamente sencilla, pero a efectos de ilustración puede servirnos para mostrar el procedimiento. En la Tabla 10 se puede observar que la correlación de los dos factores $(0,644)$ elevada al cuadrado es inferior a la varianza extraída de ambos factores, lo que significa que el factor 1 y el factor 2 están midiendo constructos diferentes.

\section{Tabla 10. Evidencias discriminantes}

\begin{tabular}{l|c|c}
\hline & Factor 1 & Factor 2 \\
\hline Factor 1 & 0,597 & \\
\hline Factor 2 & 0,414 & 0,409 \\
\hline
\end{tabular}

Una segunda evidencia sostiene que el valor de la correlación, más y menos el valor del producto de 1,96 por la desviación estándar de la covarianza del indicador, debe formar un intervalo en el que el uno no se encuentre comprendido entre los extremos (Hair et al., 1999). En nuestro caso, si a la correlación de 0,644 le sumamos y restamos el producto de 1,96 por 0,08 obtenemos el intervalo $[0,47-0,81]$ donde el 1 no se encuentra comprendido entre los extremos. Por tanto, este segundo criterio también se cumple en ambas dimensiones.

\section{DISCUSIÓN}

Extraído de un análisis de contenido de carácter exploratorio Del Junco et al. (2013) proponen una escala que inicialmente es considerada por los autores como un instrumento de la felicidad organizativa. Analizando con detenimiento el contenido de la escala, se observa que representa antecedentes de la felicidad laboral, y no mide la felicidad en sí misma. El análisis de validez de los ítems propuestos refleja que en la escala subyacen dos dimensiones: factores del puesto de trabajo y factores del trabajador, las cuales añaden al balance hedónico de experiencias vitales positivas y negativas acumuladas la consecución de objetivos personales logrados también en el trabajo (Origo \& Pagani, 2009). Al mismo tiempo, los ítems de este instrumento consideran tanto las características individuales como el efecto de los factores organizacionales, siguiendo la corriente que estudia cómo manejar el conjunto de la relación trabajador-entorno (Fisher, 2010; Warr, 2013). Esta conjunción de factores internos y externos al trabajador contribuye a enriquecer los trabajos que analizan las fuentes de felicidad, ya que según Pan y Zhou, (2013), la mayor parte de los estudios sobre la felicidad se han enfocado fundamentalmente a la relación ingresos-felicidad, más que sobre otras variables. Igualmente, Seibert, Crant y Kraimer (1999) ponen de manifiesto el interés de estudiar la relación del ámbito interno y externo a la persona para conocer su impacto en la felicidad de la organización, ámbitos que son recogidos en la escala y que muestran ser dos dimensiones distintas. Dichas dimensiones son coherentes con la visión de Singh y Aggarwal (2018) acerca de que la felicidad en el trabajo implica interacción entre las experiencias del empleado individualmente y las experiencias organizativas.

Los ítems del cuestionario recogen aspectos relevantes tratados en la literatura de felicidad laboral: la satisfacción laboral (Fisher, 2003), es un factor relevante de la felicidad en el trabajo sustentado en la psicología positiva (Proudfoot, Corr, Guest, \& Dunn, 2009; Bakker \& Oerlemans, (2011)); la motivación del puesto de trabajo ha demostrado su influencia positiva en el bienestar de las personas en la empresa (López \& Fierro, 2015); el clima organizacional tanto en la unidad de trabajo como en la compañía se confirma en la literatura como un factor relevante en la felicidad (Chiumento, 2007; Gutierrez, 1988; López, Chambel, Muñoz, \& Silva da Cunha, 2018). Igualmente, el salario surge como un ítem relevante entre las fuentes de felicidad constatadas en el cuestionario (Diener \& Biswas-Diener, 2002; Frey \& Stutzer, 2000; Fuentes \& Rojas, 2001; Hagerty \& Veenhoven, 2003; Schyns, 2002; Kenny, 2005; Zuzanek, 2013). El diseño de las tareas es considerado por Fisher (2010) entre las características para mejorar la felicidad en el trabajo, mientas que los trabajos de Likert $(1979 ; 1986)$ mostraron la influencia de dirigir bien sobre la felicidad de las personas en la organización.

\section{CONCLUSIONES}

Si en la felicidad del trabajador sólo pesasen factores del individuo como genética o factores hereditarios, poco se podría hacer por incrementar y gestionar el nivel de felicidad (Arvey, Boucharda, Segal, \& Abraham, 1989; Haybron, 2000). Este trabajo ha desarrollado el análisis de validez de una escala propuesta previamente en la literatura por Del Junco et al. (2013) para 
medir factores de felicidad en el trabajo. De los resultados se pueden extraer conclusiones tanto a nivel teórico, como a nivel metodológico y para la práctica profesional.

En el plano teórico, en línea con Linz y Semykina (2012), el análisis de validez de la escala ha revelado dos dimensiones: Factores relacionados con el puesto de trabajo, altamente relevantes a juzgar por sus valores de validez y fiabilidad; y una segunda dimensión de factores intrínsecos del trabajador. De forma semejante a Fisher (2010), que concreta en tres niveles la medición de la felicidad en el trabajo (el trabajo en sí mismo, las características contextuales del trabajo y la organización en su conjunto), la escala aquí presentada diferencia por un lado las características del entorno (que recoge tanto el trabajo como el contexto y la organización) y por otro las características del trabajador.

Metodológicamente, este trabajo proporciona una posible guía para validar escalas siguiendo un enfoque confirmatorio, tratando de ser una ayuda para aquellos investigadores que validen escalas tanto diseñadas por ellos mismos como los resultados de la aplicación de escalas extraídas de la literatura. Asimismo, Fisher (2010) hace una llamada a la necesidad de mejorar la medición de la felicidad en el trabajo, ya que las mediciones realizadas hasta ahora recogen variables relacionadas con esta en términos generales. Este trabajo trata de contribuir a la superación de esta limitación con una escala más concreta que ha mostrado cumplir los requisitos de fiabilidad y validez. Si bien, como sugieren Ones y Viswesvaran (1996), cuanto mayor es el número de variables mejores predicciones se logran, en nuestro caso el análisis estadístico demuestra que no es necesaria una gran batería de ítems.

Por último, desde un punto de vista práctico, de las 15 cuestiones propuestas por Del Junco et al. (2013), este trabajo muestra (Cuadro 4) las siguientes 11 preguntas como fiables y válidas para obtener una aproximación práctica de las fuentes de felicidad laboral de los empleados.

\section{Cuadro 4. Cuestionario aplicable en la práctica profesional para medir la felicidad en el trabajo}

\begin{tabular}{l}
\hline Evalúe del $\mathbf{1}$ al 7 las siguientes afirmaciones \\
\hline En el trabajo obtengo las justas recompensas \\
\hline El clima organizacional de la empresa es bueno \\
\hline Los jefes dirigen bien \\
\hline El clima organizacional en mi unidad de trabajo es bueno \\
\hline La motivación interna de mi puesto es alta \\
\hline Mis tareas en la empresa están bien diseñadas \\
\hline Disfruto con mi trabajo \\
\hline Tengo estabilidad interior \\
\hline Tengo bienestar objetivo \\
\hline Tengo estabilidad profesional \\
\hline Disfruto haciendo bien mi trabajo \\
\hline
\end{tabular}

La utilidad de esta sencilla escala radica en que, sin tener que invertir en consultoras o empresas especializadas, el directivo puede obtener una aproximación al nivel de felicidad de los trabajadores y actuar en consecuencia conforme a los resultados de la encuesta. Las medidas aquí propuestas se consideran medidas activas y directas, ya que requieren una fuente de información externa a la empresa. Junto a estas se pueden usar medidas de carácter pasivo o indirecto (retención del personal, rotación, absentismo, etc.) atractivas para las empresas por su inmediatez y bajo coste, puesto que utilizan fuentes de información interna, fácilmente accesibles.

En España la mayor parte de las empresas que forman su tejido industrial y comercial no tienen la capacidad necesaria para llevar a cabo un proceso de investigación profundo para determinar el nivel de felicidad en el trabajo de sus empleados. A este respecto puede resultar de la mayor utilidad para un directivo el uso de un cuestionario como el propuesto por Del Junco et al. (2013) que pueda aunar algunos de los constructos citados y pueda plasmar una imagen cercana a la situación de la felicidad laboral de su organización.

\section{Limitaciones y recomendaciones para futuras investigaciones}

El propio término "felicidad en el trabajo" se puede considerar el origen de algunas limitaciones del trabajo, debido a la ausencia de una definición unívoca y de su delimitación respecto a otros términos similares. Si bien hemos intentado mostrar las características fundamentales del problema de que el estudio parta de las aportaciones previas de autores diversos, sería necesario extraer nuevos factores del entorno y del propio trabajador de diversas disciplinas que enriquezcan y completen la escala aquí presentada. En cuanto a la validación de la escala, este trabajo no ofrece evidencias basadas en la relación con otras variables, por lo que sería conveniente que esta escala se utilizara en conjunción con medidas de la felicidad laboral que actúen como variable criterio para la validación del uso de la escala.

Respecto a la eliminación de ítems del cuestionario, esta eliminación no implica que dicho ítem no esté reflejando un factor relevante para la felicidad laboral, sino que puede obedecer a la omisión de ítems que completen una nueva dimensión relacionada con el contenido del ítem eliminado. Es lo que ha sucedido con las cuestiones relacionadas con la salud, el amor o la familia, constructos altamente complejos y relevantes según la literatura, que se han recogido en un único ítem y, al no guardar correlación con los otros, estadísticamente ha sido necesario 
omitirlo en la escala definitiva. Siguiendo con lo propuesto anteriormente, una futura línea de trabajo consistiría en ampliar el número de cuestiones relacionadas con estos factores para poder reflejar más dimensiones de un concepto tan complejo como es la felicidad laboral, incluyendo además factores que determinen positiva y negativamente el nivel de felicidad del trabajador (Ferreira et al., 2008)

Por último, vale señalar que la ubicación geográfica de la muestra (únicamente la provincia de Sevilla) puede suponer una objeción a las conclusiones de este estudio. Aunque no es probable que los factores de carácter geográfico y cultural invaliden el objeto del estudio, futuras investigaciones desarrolladas en un entorno geográfico más amplio podrían arrojar más datos sobre la fiabilidad y validez de la escala.

\section{REFERENCIAS}

American Educational Research Association, American Psychological Association, National Council on Measurement in Education. (2014). Standards for educational and psychological testing. Washington, DC: American Psychological Association.

Argyle, M. (1992). Psicología de la felicidad. Madrid, España: Alianza Editorial.

Arvey, R., Boucharda, T. Segal, N., \& Abraham, L. (1989). Job satisfaction: environmental and genetic components. Journal of Applied Psychology, 74(2), 187-192. doi:10.1037/0021-9010.74.2.187

Bagozzi, R. P., Yi, Y., \& Phillips, L. W. (1991). Assessing Construct Validity in Organizational Research. Administrative Science Quarterly, 36(3), 421-458. doi:10.2307/2393203

Baker, D., Greenberg, C., \& Hemingway, C. (2006). What happy companies know. Upper Saddle River, NJ: Pearson Prentice Hall.

Bakker, A., \& Oerlemans, W. (2011). Subjective well-being in organizations. In K. Cameron, \& G. Spreitzer (Eds.), The Oxford handbook of positive organizational scholarship (pp. 178-189). Oxford, UK: Oxford Library of Psychology.

Bentler, P. M. (1990). Comparative fit indexes in structural equation models. Psychological Bulletin, 107(2), 238-246. doi:10.1037/00332909.107.2.238

Bentler, P. M. (1992). On the fit of models to covariances and methodology to the Bulletin. Psychological Bulletin, 112(3) 400-404. doi:10.1037/0033-2909.112.3.400

Benz, M., \& Frey, B. S. (2004). Being independent raises happiness at work. Swedish Economic Policy Review, 11(2), 95-134.

Byrne, B. M. (2006). Structural equation modelling with EQS: Basic concepts, applications, and programming. Mahwah, NJ: Lawrence Erlbaum Associates Publishers.

Chiumento, S. (2007). Happiness at Work Index: Research Report 2007. London, UK: Chiumento.

Clark, A., Kristensen, N., \& Westergård-Nielsen, N. (2009). Job satisfaction and coworker wage: Status or signal? Economic Journal, 119(5), 430-447.
Curran, P. S., West, S. G., \& Finch, J. F. (1996). The robustness of test statistics to nonnormality and specification error in confirmatory factor analysis. Psychological Methods, 1(1), 16-29. doi:10.1037/1082989X.1.1.16

Daniels, K. (2000). Measures of five aspects of affective well-being at work. Human Relations, 53(2), 275-294. doi:10.1177/a010564

Del Junco, J. G., Espasandín, F., Dutschke, G. M. J., \& Palacios, B. (2013). An approach to the design of a scale for measuring happiness at work of Iberian Companies Athens. ATINER'S Conference Paper Series, No: SME2013-0855.

Diener, E. (2000). Subjective well-being: The science of happiness and a proposal for a national index. American Psychologist, 55(1), 34-43. doi:10.1037/0003-066X.55.1.34

Diener, E., Sandvik, E., \& Pavot, W. (1991). Happiness is the frequency, not the intensity, of positive versus negative affect. In F. Strack, M. Y. Argyle, N. Schwarz (Eds.), Subjective well-being: An interdisciplinary perspective (pp. 119-139). Elmsford, NY: Pergamon Press.

Diener, E., Wirtz, D., Tov, W., Kim-Prieto, C., Choi, D.-W., Oishi, S., BiswasDiener, R. (2010). New well-being measures: Short scales to assess flourishing and positive and negative feelings. Social Indicators Research, 97(2), 143-156. doi:10.1007/s11205-009-9493-y

Diener, E., Suh, E. M., Lucas, R. E., \& Smith, H. L. (1999). Subjective well-being: Three decades of progress. Psychological Bulletin, 125(2), 267-302. doi:10.1037/0033-2909.125.2.276

Diener, E., \& Biswas-Diener, P. (2002). Will money increase subjective well-being? A literature review and guide to needed research. Social Indicators Research, 57(2), 119-169.

Dillman, D. A. (2000). Mail and internet surveys: The tailored design method. New York, NY: Wiley.

Ferreira, M. C., Silva, A. P. C., Fernandes, H. D. A., \& Almeida, S. P. D. (2008). Desenvolvimento e validação de uma escala de afetos no trabalho (ESAFE). Avaliação Psicológica, 7(2) 143-150.

Fisher, C.D. (2003). Why do lay people believe that satisfaction and performance are correlated? Possible sources of commonsense theory. Journal of Organizational Behavior, 24(6), 753-777. doi:10.1002/job.219

Fisher, C. D. (2010). Happiness at work. International Journal of Management Reviews, 12(4), 384-412. doi:10.1111/j.14682370.2009.00270.x

Fisher, C. D. (2014). Conceptualizing and measuring wellbeing at work. In P. Y. Chen \& C. L. Cooper (Eds.), Work and Wellbeing (pp. 9-33). Chichester, UK: Wiley-Blackwell.

Fornell, C., \& Larcker, D. F. (1981). Evaluating structural equation models with unobservable variables and measurement error. Journal of Marketing Research, 18(1), 39-50. doi:10.2307/3151312

Frey, B., \& Stutzer, A. (2000). Subjective well-being, economy and institutions. Economic Journal, 110, 918-938.

Fuentes, N., \& Rojas, M. (2001). Economic theory and subjective well-being: Mexico. Social Indicators Research, 53(3), 289-314. doi:10.1023/A:1007189429153

Gamero, C. (2007). Satisfacción laboral y tipo de contrato en España. Investigaciones económicas, 31(3), 415-444.

George, D., \& Mallery, M. (2003). Using SPSS for Windows step by step: A simple guide and reference. Boston, MA: Allyn y Bacon. 
Groot, W., \& Maassen van den Brink, H. (1999). Job satisfaction and preference drift. Economics Letters, 63(3), 363-367. doi:10.1016/ So165-1765(99)00053-1

Gutierrez, L. H. S. (1988) Percepção do clima organizacional conforme o escalão hierárquico. RAE-Revista de Administração de Empresas, 28(4), 5-13. doi:10.1590/S0034-75901988000400002

Hagerty, M., \& Veenhoven, R. (2003). Wealth and happiness revisited: Growing national income does go with greater happiness. Social Indicators Research, 64(1), 1-27.

Hair, J. F. Jr., Anderson, R. E., Tatham, R. L., \& Black, W. C. (1999). Análisis Multivariante, $5^{\text {a }}$ edición. Madrid, España: Prentice Hall Iberia.

Haybron, D. M. (2000). Two philosophical problems in the study of happiness. Journal of Happiness Studies, 1(2), 207-225. doi:10.1023/A:1010075527517

Heller, D., Judge, T., \& Watson, D. (2002). The confounding role of personality and trait affectivity in the relationship between job and life satisfaction. Journal of Organizational Behaviour, 23(7), 815-835. doi:10.1002/job.168

Hosie, P. J., \& Sevastos, P. (2009). Does the "happy-productive worker" thesis apply to managers?. International Journal of Workplace Health Management, 2(2), 131-160. doi:10.1108/17538350910970219

Hu, L. T., \& Bentler, P. (1999). Cutoff criteria for fit indices in covariance structure analysis: Conventional criteria versus new alternatives. Structural Equation Modeling, 6(1), 1-55. doi:10.1080/10705519909540118

Hu, L.T., Bentler, P. M., \& Kano, Y. (1992). Can test statistics in covariance structure analysis be trusted? Psychological Bulletin, 112(2), 351-362. doi:10.1037/0033-2909.112.2.351

Huppert, F. A., \& So, T. T. C. (2013). Flourishing across Europe: Application of a new conceptual framework for defining well-being. Social Indicators Research, 110(3), 837-861. doi:10.1007/s11205-0119966-7

Judge, T. A., Heller, D., \& Mount, M. K. (2002). Five-factor model of personality and job satisfaction: A meta-analysis. Journal of applied psychology, 87(3), 530-541. doi:10.1037/0021-9010.87.3.530

Judge, T. A., Piccolo, R. F., Podsakoff, N. P., Shaw, J. C., \& Rich, B. L. (2010). The relationship between pay and job satisfaction: A meta-analysis of the literature. Journal of Vocational Behavior, 77(2), 157-167.

Kenny, C. (2005). Does development make you happy? Subjective wellbeing and economic growth in developing countries. Social Indicators Research, 73(2), 199-219. doi:10.1007/511205-004-0986-4

Keyes, C. L. M. (2000). Subjective change and its consequences for well-being. Motivation and Emotion, 24(2) , 67-84. doi:10.1023/A:1005659114155

Keyes, C. L. M. (2002). The mental health continuum: From languishing to flourishing in life. Journal of health and social behavior, 43(2) 207222. doi:10.2307/3090197

Kiesebir, P., \& Diener, E. (2008). In pursuit of happiness: empirical answers to philosophical questions. Perspective and Psychological Science, 3(2), 117-125. doi:10.1111/j.1745-6916.2008.00069.x

Likert, R. (1979). From production and employee to system 1-4. Journal of Management, 5(2), 147-156. doi:10.1177/014920637900500205

Likert, R. (1986). Nuevas formas de solucionar conflictos. México. Editorial Trillas. (Versión original: New Way of managing conflict, 1975).
Linz, S. J., \& Semykina, A. (2012). What makes workers happy? Anticipated rewards and job satisfaction. Industrial Relations: $A$ Journal of Economy and Society, 51(4), 811-844. doi:10.1111/j.1468232X.2012.00702.X

López, J., \& Fierro, I. (2015). Determinantes de la felicidad en los administradores: Una investigación realizada en las farmacias del Grupo Difare en Ecuador. Universidad \& Empresa, 17(29), 181-211. doi:10.12804/rev.univ.empresa.29.2015.08

López, S., Chambel, M. J., Muñoz, F., \& Silva da Cunha, B. (2018). The role of perceived organizational support in job insecurity and performance. RAE-Revista de Administração de Empresas, 58(4), 393404. doi:10.1590/So034-759020180405

Luthans, F. (2002). The need for and meaning of positive organizational behavior. Journal of organizational behavior, 23(6), 695-706. doi:10.1002/job.165

Moyano Díaz, E., Castillo Guevara, R., \& Lizana Lizana, J. (2008). Trabajo informal: Motivos, bienestar subjetivo, salud, y felicidad en vendedores ambulantes. Psicologia em Estudo, 13(4), 693-701. doi:10.1590/S1413-73722008000400007

Onusic, L. M. (2013). Determinantes e antecedentes do bem-estar e da felicidade. RAE-Revista de Administração de Empresas, 53(4), 418 418. doi:10.159o/So034-75902013000400010

Origo, F., \& Pagani, L. (2009). Flexicurity and job satisfaction in Europe: The importance of perceived and actual job stability for well-being at work. Labour economics, 16(5), 547-555. doi:10.1016/j. labeco.2009.02.003

Pan, J., \& Zhou, W. (2013). Can success lead to happiness? The moderators between career success and happiness. Asia Pacific Journal of Human Resources, 51(1), 63-80. doi:10.1111/j.17447941.2012.00033.x

Parker, G. B., \& Hyett, M. P. (2011). Measurement of well-being in the workplace: The development of the Work Well-Being Questionnaire. The Journal of nervous and mental disease, 199(6), 394-397. doi:10.1097/NMD.obo13e31821cd3b9

Paschoal, T., \& Tamayo, A. (2008). Construção e validação da escala de bem-estar no trabalho. Avaliação Psicológica, 7(1), 11-22.

Peterson, C., Park, N., Hall, N., \& Seligman, M. E. (2009). Zest and work. Journal of Organizational Behavior, 30(2), 161-172. doi:10.1002/ job. 584

Proudfoot, J., Corr, P., Guest, D., \& Dunn, G. (2009). Cognitive-behavioral training to change attributional style improves employee well-being, job satisfaction, productivity, and turnover. Personality \& Individual Differences, 46(2), 147-153. doi:10.1016/j.paid.2008.09.018

Rothmann, S. (2013). From happiness to flourishing at work: A Southern African perspective. In M. Wissing (Eds), Well-being research in South Africa: Cross-cultural advances in positive psychology (pp. 123151). Dordrecht, Netherlands: Springer.

Ryan, R. M., \& Deci, E. R. (2001). On happiness and human potentials: A review of research on hedonic and eudaimonic well-being. Annual Review of Psychology, 52, 141-166. doi:10.1146/annurev. psych.52.1.141

Sánchez, M., \& Sarabia, F. J. (1999). Validez y fiabilidad de escalas. In S. Sánchez (Coord.), Metodología para la investigación en marketing y dirección de empresas (pp. 363-393). Madrid, España: Ediciones Pirámide. 
Sanín, A., \& Restrepo, M. (2009). Vinculación laboral, motivación y desempeño de empleados de carrera y supernumerarios en una universidad del estado. Revista Interamericana de Psicología Ocupacional, 28(2), 149-183.

Sanín, A., López, A., \& Gómez, B. (2015) “. Los efectos del tipo de contrato laboral sobre la felicidad en el trabajo, el optimismo y el pesimismo laborales. Revista Interamericana de Psicología Ocupacional, 34(1), 9-21. doi:10.21772/ripo.v34n1ao1

Satorra, A. (1993), Asymptotic robust inferences in multi-sample analysis of augmented-moment matrices. In C. R. Rao, \& C. M. Cuadras (Eds.), Multivariate analysis, future directions (pp. 211-229). Amsterdam, Holland.

Schyns, P. (2002). Wealth of nations, individual income and life satisfaction in 42 countries: A multilevel approach. Social Indicators Research, 6o(1-3), pp 5-40. doi:10.1023/A:1021244511064

Seibert, S. E., Crant, J. M., \& Kraimer, M. L. (1999). Proactive personality and career success. Journal of Applied Psychology, 84(3), 416-427. doi:10.1037/0021-9010.84.3.416

Seligman, M. E., \& Csikszentmihalyi, M. (2000). Positive psychology: An introduction. American Psychologist, 55(1), 5-14. doi:10.1037/0003066X.55.1.5

Singh, S., \& Aggarwal, Y. (2018). Happiness at work scale: Construction and psychometric validation of a measure using mixed method approach. Journal of Happiness Studies, 19(5), 1439-1463. doi:10.1007/s10902-017-9882-X

Sloane, P., \& Williams, H. (2000). Job satisfaction, comparison earnings, and gender. Labour Review, 14(3), 473-502. doi:10.1111/14679914.00142

Suh, E., \& Koo, J. (2008). Comparing subjective wellbeing across cultures and nations. What and why questions. In M. Eid, \& R. Larsen (Eds.), The Science of subjective well-being (pp. 414-427), New York, NY: The Guilford Press.
Tanaka, J. S. (1993). Multifaceted conceptions of fit in structural equation models. In K.A. Bollen, \& J. S. Long (Eds.), Testing structural equation models (pp. 10-39), Newbury Park, CA: Sage Focus Edition.

Useche, M. C., \& Parra, B. Q. (2002). La organización del trabajo en el marco de la globalización. Revista Gaceta Laboral, 8(1), 67-78.

Valentini, F., \& Damásio, B. F. (2017). Variância média extraída e confiabilidade composta: Indicadores de precisão. Psicologia: Teoria e Pesquisa, 32(2), e322225. doi:10.1590/0102-3772e322225

Van Horn, J. E., Taris, T. W., Schaufeli, W. B., \& Schreurs, P. J. G. (2004). The structure of occupational well-being: A study among Dutch teachers. Journal of Occupational and Organizational Psychology, 77(3), 365-375. doi:10.1348/0963179041752718

Veenhoven, R. (1991). Is happiness relative? Social Indicators Research, 24(1), 1-34. doi:10.1007/BFoo292648

Warr, P. B. (1994). A conceptual framework for the study of work and mental health. Work and Stress, 8(2), 84-97. doi:10.1080/02678379408259982

Warr, P. B. (2013). Fuentes de felicidad e infelicidad en el trabajo: Una perspectiva combinada. Revista de Psicología del Trabajo y de las Organizaciones, 29(3), 99-106. doi:10.5093/tr2013a15

Wright, T., Larwood, L., \& Denney, P. (2002). The different 'faces' of happiness - unhappiness in organizational research: Emotional exhaustion, positive affectivity, negative affectivity, and psychological. Journal of Business and Management, 8, 34-51.

Zelenski, J. M., Murphy, S. A., \& Jenkins, D. A. (2008). The happyproductive worker thesis revisited. Journal of Happiness Studies, 9(4), 521-537. doi:10.1007/s10902-008-9087-4

Zuzanek, J. (2013). Does being well-off make us happier? Problems of measurement. Journal Happiness Study, 14(3), 795-815. doi:10.1007/ s10902-012-9356-0 